\title{
Compound Composite Odontoma in Mandibular Symphysis - A Rare Case
}

\author{
Sushrut Vaidya ${ }^{1}$, Usha Asnani $^{2}$, Smita Sonavane ${ }^{3}$, Imran Khalid ${ }^{4}$, Kartik Poonja ${ }^{5}$, Alok Bhardwaj ${ }^{6}$
}

\begin{abstract}
Compound odontomas are considered as hamartomatous malformation rather than true neoplasms $\&$ are generally asymptomatic. The exact etiology is unknown and is often associated with the overretained deciduous teeth, most commonly in maxillary anterior region. In this case, multiple denticles or rudimentary teeth numbering 42 were enucleated from the mandibular symphysis region of $17 \mathrm{yr}$ old female which makes this case rare and unusual. Evidence of concrescence, fusion, dilaceration were observed in the denticles enucleated, the size of which ranged from $2 \mathrm{~mm}$ to $10 \mathrm{~mm}$.
\end{abstract}

Key Words: Odontoma, Compound Odontoma, Denticles, Hybrid Odontoma, Impacted Teeth

\section{Introduction}

Odontomas are considered as developmental anomalies arising from completely differentiated epithelial and mesenchymal cells that give rise to ameloblast \& odontoblast. They are hamartomatous lesions rather than true neoplasms. ${ }^{1}$ The term 'odontoma' was coined by Paul Broca in 1867 which by definition alone refers to any tumour of odontogenic origin. Most of the odontomas are asymptomatic, although some signs \& symptoms relating to their presence may occur. The compound composite odontomas are a malformation in which all the dental tissues are in a more orderly pattern than in the complex odontoma so that the lesion may consist of many tooth like structures. ${ }^{2}$ Compound odontomas are generally most commonly seen in maxillary anterior region with denticles varying from 4-28. ${ }^{3}$ The sheer number of denticles extracted

\section{Reader $^{1}$}

Professor ${ }^{2}$

Reader $^{3}$

Lecturer $^{4}$

Post graduate ${ }^{5}$

Post graduate ${ }^{6}$

Dept Oral and Maxillofacial Surgery,

MGM Dental College and Hospital, Navi Mumbai.

Address for Correspondence:

Dr. Sushrut Vaidya,

Reader

Dept Oral and Maxillofacial Surgery,

MGM Dental College and Hospital, Navi Mumbai.

Mob.: 9869160530

E-mail: ezeesush79@gmail.com numbering 42 from the mandibular symphysis region makes the case a rare and unique one.

\section{Case History}

A $17 \mathrm{yr}$ old female presented with chief complaint of malaligned mandibular anterior teeth (Fig.1). Patient was asymptomatic without any specific complaint. On examination she had over retained lower deciduous left central incisor, partially erupted and medially tipped lower left permanent lateral incisor with missing canine. The mandibular right central incisor was tipped labially. Patient was advised orthopantamogram for further treatment. OPG showed multiple tooth like structures of different size and shape in relation to root of mandibular anterior teeth in the symphysis region which was surrounded by circumscribed radiolucent zone. There was displacement of permanent right mandibular central incisor(Fig.2). The left mandibular lateral incisor was displaced and the canine was impacted apical to the premolars. Upon the clinical and radiographic findings a provisional diagnosis of compound composite odontoma was made. It was

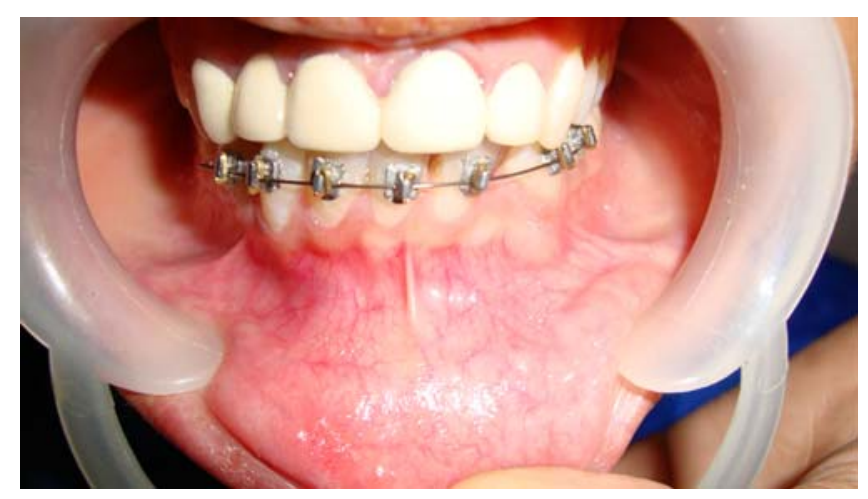

Fig.1: Preoperative intraoral photograph 


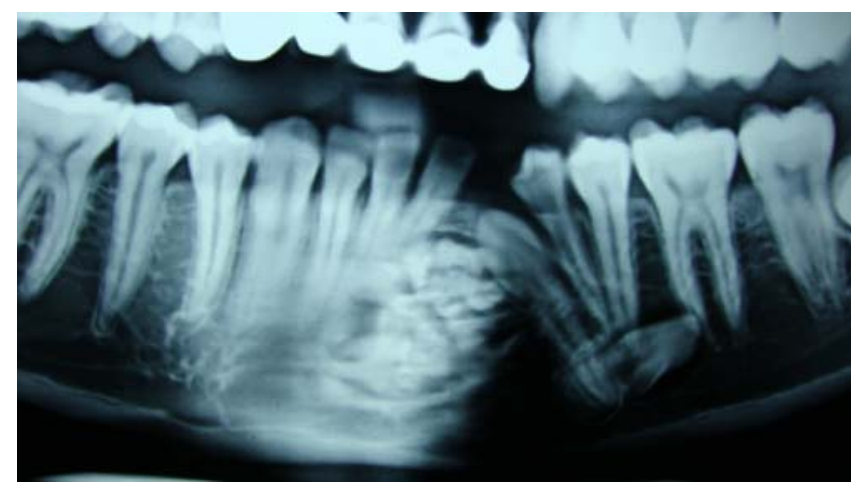

Fig.2 : Preoperative radiograph showing the denticles

decided to extract the deciduous central incisor and surgically enucleate the tumour. Under local anaesthesia deciduous central incisor was extracted. A labial mucoperiosteal flap was raised. The bone covering the odontoma was removed and (Fig.3) numerous denticles around 42 were enucleated along with the capsule. Evidence of concrescence were seen

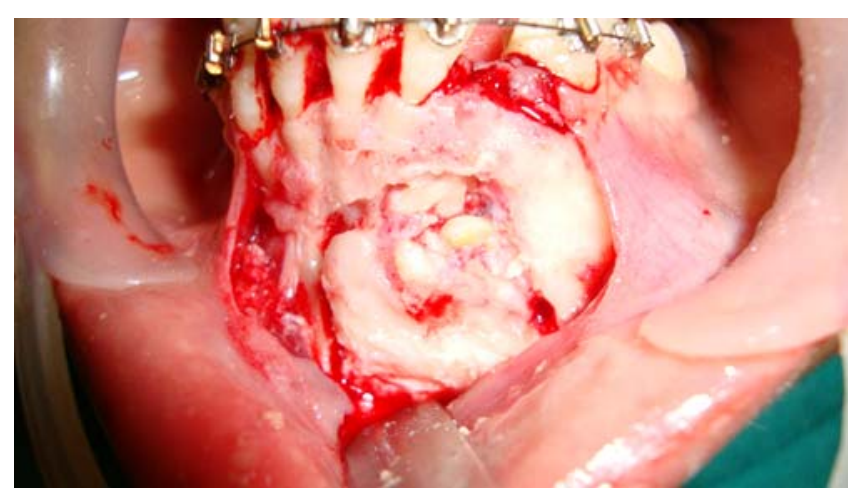

Fig.3 : Mucoperiosteal flap raised showing the lesion

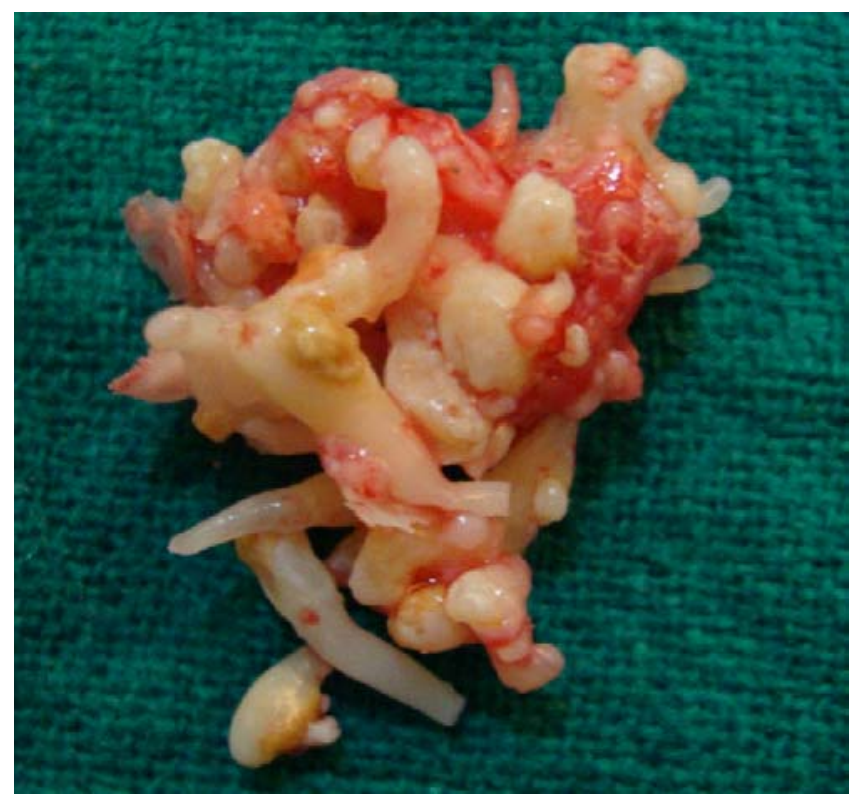

Fig. 4 : Specimen after excision

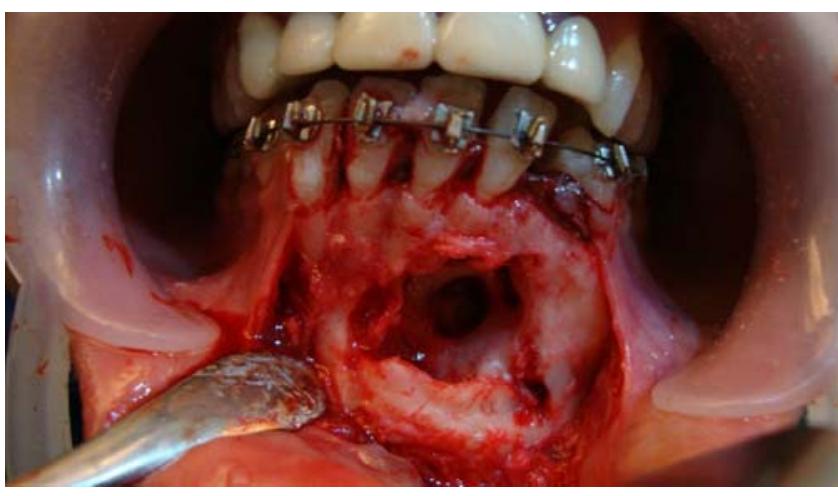

Fig.5 : Postoperative bony defect seen

in 4 groups of denticles. Fusion and dilaceration were also noticed (Fig.4). Size of denticles varied from $4 \mathrm{~mm}$ to $10 \mathrm{~mm}$. The partially erupted and displaced lateral incisor and impacted canine were left in place. Closure was done with 3-0 vicryl. Post operative period was uneventful.

\section{Discussion}

The term odontoma by definition refers to a benign, mixed, calcified tumour of odontogenic origin. The absolute incidence of odontogenic tumours varies from $0.002 \%$ to $0.1 \%{ }^{4}$ out of which odontomas constitute about $22 \%,{ }^{5}$ of which $10 \%$ are compound odontomas.

There are essentially 2 types of odontomas:

1) Complex composite odontoma

2) Compound composite odontoma

As per the WHO classification complex composite odontoma is defined as a malformation in which all dental tissues are well formed but are arranged in disorderly pattern.

Compound composite odontoma is a malformation in which all dental tissues are represented in a more orderly pattern than in the complex odontomas, so that the lesion contains tooth like rudimentary structures in which each enamel, dentin, cementum and pulp are arranged as in normal teeth.

The exact etiology of odontoma is not known ${ }^{6}$ yet cases have related odontoma to local trauma, infection and genetics. It arises from an exubrant proliferation of dental lamina or its remnants and is termed as laminar odontome, or forms as a result of multiple schizontia i.e a locally conditioned hyperactivity of dental lamina. ${ }^{7}$ It may be associated with Gardners syndrome of intestinal polyposis. ${ }^{8}$ Compound odontomas are twice as commonly observed as complex and commonly in the maxillary anterior region. Clinically odontomas usually remain small rarely exceeding the diameter of the teeth. It may become large and causes expansion of cortical bone most commonly when associated with 
dentigerous cyst. They usually present with unerupted or impacted teeth or retained deciduous teeth. ${ }^{9}$ Despite interference with eruption there is no resorption of adjacent tooth root. There is often displacement of the adjacent tooth with loss of vitality.

Radiographically odontomas appear as an irregular radioopacity or denticles surrounded by a radiolucency with or without bony expansion.

Odontomas are successfully treated by surgical enucleation with least rate of recurrence. Kaban stated that odontomas are easily enucleated and adjacent teeth that may have been displaced by lesion are seldom harmed by surgical excision because they are usually separated by the septum of bone. ${ }^{10}$

Although odontomas are considered as hamar-tomatous lesions having a limited growth potential, there are chances of undergoing secondary changes like dentigerous cyst formation, ameloblastic transformation, causing weaking of the surrounding bone. Hence on detection, surgical enucleation of odontomas, followed by curettage is recommended to prevent further complication.

\section{References}

1. Budnick, S., 1976. Compound and complex odontomas. Oral Surg. Oral Med. Oral Pathol. 42, 501-506.

2. Philipsen, H.P., Reichart, P.A., Praetorious, F., 1997. Mixed odontogenic tumours and odontomas. Considerations on interrelationship.Review of literature and presentation of 134 new cases of odontomas. Oral Oncol. 33, 86-99.

3. Amado-Cuesta, S., Gargallo-Albiol, J., Berini-Aytes, L., Gay-Escoda, C., 2003. Review of 61 cases of odontoma. Presentation of an erupted complex odontoma. Med. Oral. 8, 366-373.

4. Yeung, K.H., Cheung, R.C.T., Tsang, M.M.H., 2003. Compound odontoma associated with an unerupted and dilacerated maxillary primary central incisor in a young patient. Int. J. Ped. Dent. 13, 208-212.

5. Saadettin, Dagistan, Mustafa, Goregen, Ozkan, Miloglu, 2007.Compound odontoma associated with maxillary impacted permanent central incisor tooth: a case report: the internet. J. Dent. Sci. 5,1-6.

6. Shafer, Hine, Levy, 1993. A Textbook of Oral Pathology, fourth ed. W.B. Saunders \& Co., pp. 308-312.

7. Philipsen HP, Reichart PA, Praetorious F. Mixed Odontogenic Tumours and Odontomas. Considerations on Interrelationship. Review of Literature and Presentation of 134 New Cases of Odontomas. Oral Oncol1997;33:8689.

8. Cawson, R.A., Binnie, W.H., Barrett, A.W., Wright, J.M., 2001. Oral Disease, third ed. Mosby, p. 6.24.

9. Singh, S., Singh, M., Singh, I., Khandelwal, D., 2005. Compound composite odontome associated with an unerupted deciduous incisor - a rarity. J. Ind. Soc. Ped. Prev. Dent. 23, 146-150.

10. Kaban LB.Pediatric Oral and Maxillofacial surgery Philadelphia: Saunders; 1990.p.111-2. 